\title{
Retroperitoneal fibrosis - a report of five cases
}

\author{
Marta Runowska, Dominik Majewski, Mariusz Puszczewicz \\ Department of Rheumatology and Internal Diseases, Poznan University of Medical Science, Poland
}

\begin{abstract}
Retroperitoneal fibrosis (RPF) is a rare disease, characterized by inflammation and deposition of fibrotic tissue in the vicinity of the abdominal aorta and iliac arteries. We present a report of five patients admitted to our department between January 2014 and February 2017, diagnosed with RPF. Abdominal pain was the most common presenting symptom; however, in one patient, RPF was identified accidentally in routinely performed ultrasonography. In 4 cases, corticosteroids (CS) in combination with azathioprine were applied as first-line therapy, whereas one patient was treated with intravenous methylprednisolone pulses followed by oral CS. In this paper, clinical features as well as laboratory and radiographic findings together with management and treatment outcomes in patients with RPF are discussed. Given the rarity of the condition, it seems important to report every single case of RPF to help establish its management algorithm.
\end{abstract}

Key words: corticosteroids, azathioprine, retroperitoneal fibrosis, Ormond's disease.

\section{Introduction}

Retroperitoneal fibrosis (RPF) is a fibroinflammatory condition of still not fully known pathogenesis. Its origin is idiopathic in about 2/3 of cases (idiopathic retroperitoneal fibrosis, IRF), while the remaining one third arise secondary to a number of factors, e.g. malignancies, infections, drugs, surgery, injuries [1]. Differentiation between these two forms is crucial to determine the therapeutic strategy, because in the secondary forms, it is aimed at the removal of an underlying cause. Cross-sectional imaging techniques, such as computed tomography (CT) and magnetic resonance imaging (MRI), are the modalities of choice in diagnosis of RPF. However, in any atypical manifestation, especially in cases which raise the suspicion of underlying malignancy, the biopsy of retroperitoneal tissue is mandatory to establish the diagnosis. There are no universal guidelines for the treatment of IRF, due to its rarity and to the lack of randomized controlled trials comparing different therapeutic strategies. However, based on current literature, corticosteroids (CS) remain the therapy of choice in inducing remission. Despite the high success rates, relapses after discontinuation of CS are reported in 24 to $72 \%$ of cas- es [2]. Hence, attempts are made to combine CS with other immunosuppressant agents, but the outcomes are mostly presented as case reports, small case series or uncontrolled trials.

\section{Case report}

We reviewed the medical records of all consecutive patients with retroperitoneal fibrosis who were admitted to our department from January 2014 to February 2017. All of the patients were men and the median age at diagnosis was 53 (range 26 to 64 years). The most common comorbidities included hypertension (80\%), dyslipidaemia (80\%) and diabetes mellitus (60\%). Two patients (40\%) had a positive smoking history. Four patients (80\%) were symptomatic, with pain as the most common presenting symptom. The pain was located in the abdomen in three cases and in the loins in the remaining one. In two patients, it was described as non-specific and dull, whereas in two cases it had colic character. Constitutional symptoms (fatigue, weight loss) were present in two cases. Two patients presented with renovascular hypertension and one of them developed hypertensive crisis and acute post-renal kidney injury. The duration of symptoms from

Address for correspondence:

Marta Runowska, Department of Rheumatology and Internal Diseases, Poznan University of Medical Science, 28 Czerwca 1956 r. 135/147, 61-545 Poznan, Poland, e-mail: marta.runowska1@gmail.com

Submitted: 3.04.2017; Accepted: 22.05.2017 
onset to diagnosis ranged from one to several months. One patient was asymptomatic and bilateral hydronephrosis was detected accidentally in routinely performed abdominal ultrasonography.

In all patients, the initial diagnosis of RPF was established in imaging studies, namely CT and/or MRI. Soft tissue density surrounding the abdominal aorta was the most common finding, as seen in 4 cases (80\%), followed by perirenal location of the mass in one case. Abdominal aortic aneurysms were found in none of our patients. Hydronephrosis was present in $3(60 \%)$ cases, among which in two it had bilateral character. One patient required CT-guided needle biopsy to rule out a suspected malignancy, due to the atypical RPF localization. However, the histopathologic findings of the specimens were nonspecific and included fragments of connective tissue and skeletal muscle.

The baseline laboratory results, before initiation of immunosuppressive treatment, were available in three patients. Two patients had mild anaemia and elevated concentration of C-reactive protein. None of the patients with available serum creatinine measurements had renal failure. Demographic and clinical characteristics of all 5 patients are presented in Table I.
The serum samples of our patients were assayed for the presence of antinuclear antibodies (ANA), antineutrophil cytoplasmic antibodies (ANCA), anti-cyclic citrullinated peptide (ACPA) and rheumatoid factor (RF). Low-titre ANA were detected in three patients, one patient was positive for c-ANCA (titre 1/640, by indirect immunofluorescence) and one was positive for RF. In two cases, antibodies against Yersinia were assessed and were found positive. None of the patients had evidence of a concomitant autoimmune disorder. A summary of serological findings of all patients is provided in Table II.

During the first hospitalization, differential diagnosis between idiopathic and secondary forms of RPF was conducted, including malignancies, infections, intake of certain drugs, etc. One patient had a history of vesical tumour, one patient had previous abdominal surgery and three patients used $\beta$-blockers. Two patients had a positive QuantiFERON test, but underlying tuberculosis was excluded. One patient, due to the atypical RPF location and to the presence of monoclonal protein $M$ in the serum, was suspected of having a haematological condition. Eventually, the patient was diagnosed with monoclonal gammopathy of undetermined significance (MGUS).

Table I. Demographic and clinical characteristics of the patients with retroperitoneal fibrosis

\begin{tabular}{|c|c|c|c|c|c|}
\hline Patient No. & 1 & 2 & 3 & 4 & 5 \\
\hline Age (years)/Gender & $26 / M$ & $59 / M$ & $56 / M$ & $64 / M$ & $60 / M$ \\
\hline Presenting symptoms & $\begin{array}{l}\text { Colic-like pain, } \\
\text { hypertension }\end{array}$ & $\begin{array}{l}\text { Lower limbs } \\
\text { swelling, fatigue, } \\
\text { dull flank pain, } \\
\text { hypertensive } \\
\text { crisis }\end{array}$ & Asymptomatic & $\begin{array}{l}\text { Abdominal pain } \\
\text { radiating to the } \\
\text { groin }\end{array}$ & $\begin{array}{l}\text { Right-sided col- } \\
\text { icky pain, weight } \\
\text { loss }\end{array}$ \\
\hline Ureteral obstruction & Right & Bilateral & Bilateral & No & No \\
\hline Smoking history & No & Current & Former & No & No \\
\hline Biopsy performed & No & No & Yes & No & No \\
\hline Baseline CRP (mg/l) & - & - & - & 58.7 & 18,58 \\
\hline Baseline $\mathrm{Hb}(\mathrm{g} / \mathrm{dl})$ & 14.34 & - & - & 11.4 & 11.7 \\
\hline $\begin{array}{l}\text { Baseline sCr }(\mathrm{mg} / \mathrm{dl}) \text { or } \\
\text { GFR (ml/min) }\end{array}$ & 1.22 & - & - & $G F R=90$ & 1.15 \\
\hline
\end{tabular}

CRP - C-reactive protein; $\mathrm{Hb}$ - haemoglobin; $\mathrm{SCr}$ - serum creatinine; GFR - glomerular filtration rate; $M$ - male

Table II. Serological findings of the patients with retroperitoneal fibrosis

\begin{tabular}{|lccccc|}
\hline Patient No. & ANA & ANCA & RF & ACPA & Yersinia \\
\hline 1 & $1 / 80$, "midbody" & Negative & Negative & - & - \\
\hline 2 & $1 / 160$, speckled & Negative & Negative & Negative & $\operatorname{lgA}(+), \operatorname{lgG}(+)$ \\
\hline 3 & Negative & - & Negative & Negative & $\operatorname{lgM}(+), \operatorname{lgG}(+)$ \\
\hline 4 & Negative & c-ANCA, 1:640 & Positive & - & - \\
\hline 5 & $\begin{array}{c}1 / 80, \text { speckled and } \\
\text { homogenous }\end{array}$ & p-ANCA, 1:10 & Negative & - & - \\
\hline
\end{tabular}

ANA - antinuclear antibodies; ANCA - antineutrophil cytoplasmic antibodies; RF-rheumatoid factor; ACPA - anti-cyclic citrullinated peptide; Yersinia-anti-Yersinia antibodies 
The CS therapy was initiated at the time of diagnosis in four cases. In the asymptomatic patient, the immunosuppressive therapy was applied after oneyear observation, due to the progression of fibrosis in repeated CT scans. The initial CS dose, in conversion to prednisone, ranged between 0.1 and $0.6 \mathrm{mg} / \mathrm{kg} /$ daily. Four patients were treated concurrently with AZA (50 mg twice daily), whereas one patient was additionally given intravenous methylprednisolone pulses (500 mg for 3 days). In one patient, AZA was discontinued after 8 months of treatment due to intolerance (nausea, vomiting), while CS were gradually tapered to 0 within about 12 months.

With regard to surgical treatment, one patient required an urologic intervention at diagnosis, due to right-sided hydronephrosis. A laparoscopic ureterolysis was performed simultaneously with initiation of CS therapy, which resulted in a rapid relief of symptoms and normalisation of blood pressure values.
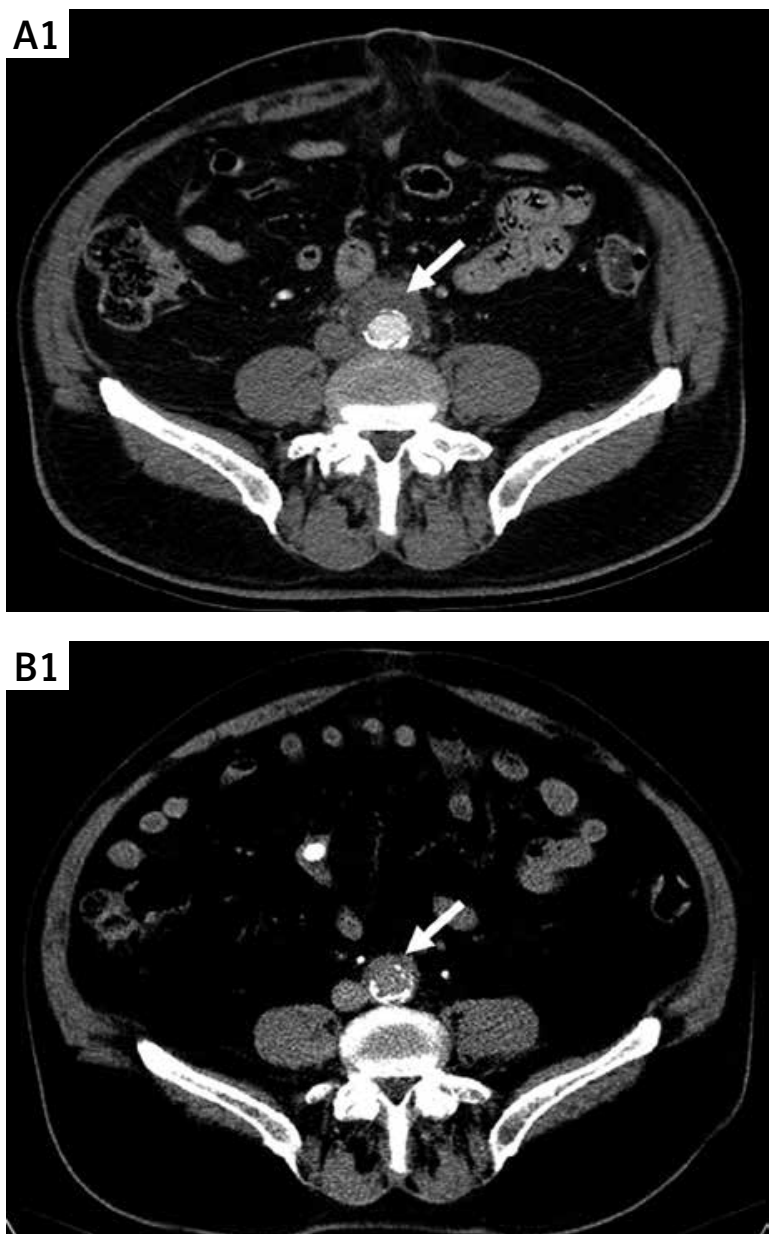

The longest follow-up in our patients lasted 27 months. Improvement, defined as resolution of symptoms, was reported within a few weeks after initiation of systemic treatment in four cases. One patient remained symptomatic after 2 months of therapy, and during the first evaluation at our department required an increase of the oral CS dose and addition of intravenous pulses. At the last follow-up visit, serum creatinine levels were normal in all patients, whereas ESR and CRP were elevated in two cases. Repeated control CT and/or MR scans showed significant (although not complete) regression of the retroperitoneal mass in four cases. In the remaining case, the first evaluation in CT has been planned at 6 months of follow-up. An example of an abdominal CT scan showing regression of fibrosis in one of our patients is presented in Figure 1. At the last observation, only one out of five patients had discontinued his therapy. The treatment strategies and outcomes of all patients are summarized in Table III.
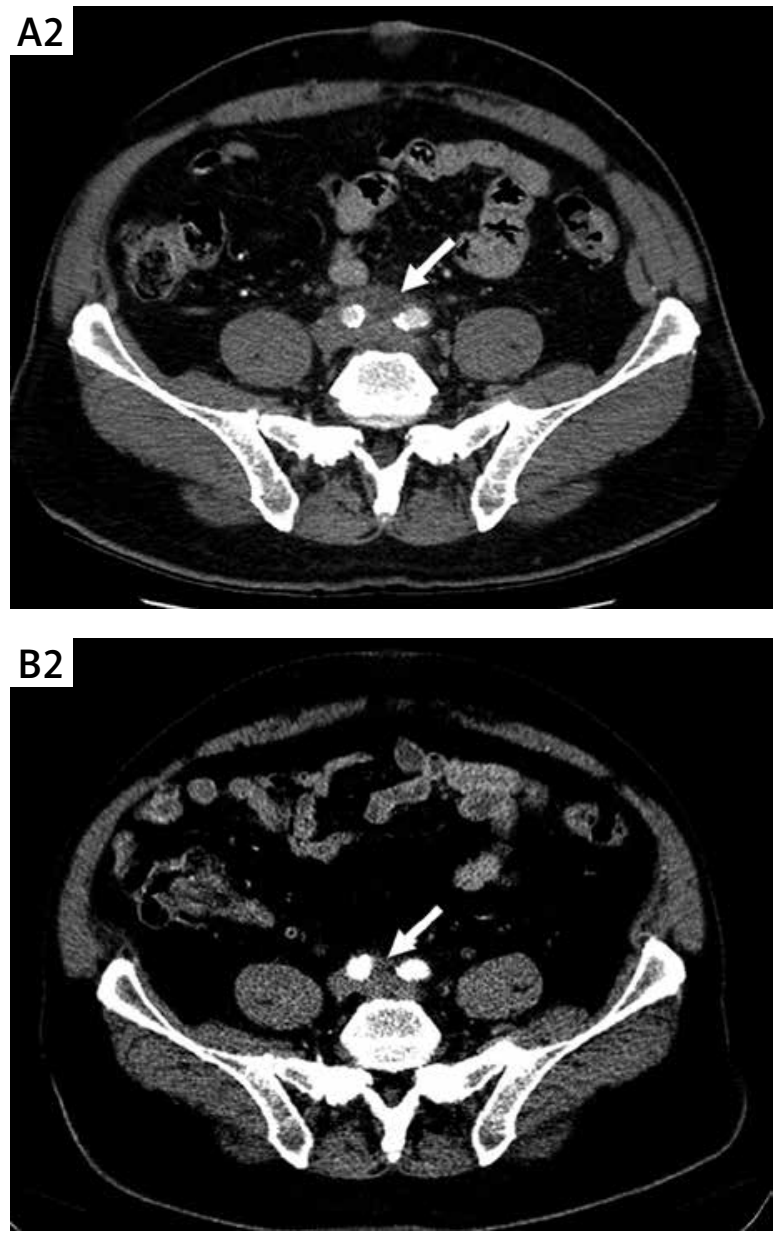

Fig. 1. Contrast-enhanced CT scan of the abdomen in patient 4 shows a retroperitoneal soft-tissue mass (arrow) of maximal $10 \mathrm{~mm}$ thickness enveloping the infrarenal aorta (A1) and proximal iliac arteries (A2). (B1, B2) Follow-up CT scan in the same patient after 5 months of treatment shows significant regression of the mass, with maximal $4 \mathrm{~mm}$ thickness. 
Table III. Modality of treatment, time of follow-up and outcomes of 5 patients with retroperitoneal fibrosis

\begin{tabular}{|c|c|c|c|c|c|}
\hline Patient No. & 1 & 2 & 3 & 4 & 5 \\
\hline Modality of treatment & $\begin{array}{l}\mathrm{CS}+\mathrm{AZA}+ \\
\text { ureterolysis }\end{array}$ & $C S+A Z A$ & $\begin{array}{c}C S+A Z A \\
\text { (discontinued) }\end{array}$ & $C S+A Z A$ & $C S+i C S$ \\
\hline $\begin{array}{l}\text { Initial CS dose in conversion } \\
\text { to prednisone (mg/kg/day) }\end{array}$ & 0.3 & 0.3 & 0.6 & 0.25 & 0.1 \\
\hline Time of follow-up (months) & 8 & 27 & 21 & 5 & 2 \\
\hline Initial symptoms & $\begin{array}{l}\text { Colic-like pain, } \\
\text { hypertension }\end{array}$ & $\begin{array}{l}\text { Lower limbs } \\
\text { swelling, fatigue, } \\
\text { dull flank pain, } \\
\text { hypertensive } \\
\text { crisis }\end{array}$ & Asymptomatic & $\begin{array}{l}\text { Abdominal pain } \\
\text { radiating to the } \\
\text { groin }\end{array}$ & $\begin{array}{l}\text { Right-sided col- } \\
\text { icky pain, weight } \\
\text { loss }\end{array}$ \\
\hline Symptoms at last follow-up & Hypertension & Asymptomatic & Asymptomatic & Asymptomatic & $\begin{array}{l}\text { Right-sided } \\
\text { abdominal and } \\
\text { lumbar pain }\end{array}$ \\
\hline Last CRP (mg/l) & 0.0 & 3.4 & 8.0 & 1.6 & 23.3 \\
\hline Last $\mathrm{Hb}(\mathrm{g} / \mathrm{dl})$ & 15.1 & 16.5 & 15.7 & 14.6 & 12.8 \\
\hline Last sCr (mg/dl) & 1.01 & 1.03 & 0.86 & 0.96 & 1.13 \\
\hline
\end{tabular}

CS - oral corticosteroid; iCS - intravenous corticosteroid; AZA - azathioprine; CRP-C-reactive protein; Hb-haemoglobin; sCr-serum creatinine

\section{Discussion}

In our study, we identified five patients diagnosed with RPF. All patients were men and the median age at diagnosis was 53, which is consistent with the demographic data of the majority of studies, suggesting that RPF affects predominantly men between 50 and 60 years of age [3]. However, one of our patients stood out among the others with his age of 26 years at presentation. It should be taken into consideration that RPF can occur at any age, and reports of this condition in children or in the elderly are not uncommon $[1,4]$. Abdominal or lumbar pain, which is thought to be the most frequent symptom of the disease [1], was the chief presenting complaint in $80 \%$ of patients in our study. Only one patient remained asymptomatic despite the presence of bilateral hydronephrosis. The clinical manifestations of RPF are generally nonspecific and heterogeneous, which makes the diagnosis of this rare condition even more challenging [5].

It should be taken into consideration that ureteral obstruction causing acute or chronic renal insufficiency is still the most common and severe complication of IRF, with $80-100 \%$ of patients showing ureteral involvement [6]. Ureteral obstruction was observed in $60 \%$ of patients. One patient had acute renal failure, but his renal function improved after initiation of treatment. None of the patients developed chronic renal failure, and their creatinine levels remained normal at the last observation. However, the duration of follow-up is too short to allow conclusions regarding renal outcomes of our patients.
The pathogenesis of IRF remains unclear. However, the disease has several clinico-laboratory features suggesting its systemic, autoimmune origin, such as the presence of constitutional symptoms, elevated concentrations of inflammatory markers, often positive autoantibodies (especially ANA) and frequent concomitance of other autoimmune conditions, such as autoimmune thyroiditis, ANCA-positive glomerulonephritis, rheumatoid arthritis, psoriasis or systemic lupus erythematosus [1,7]. In our group, three patients were ANA positive, one was positive for RF and one was highly positive for C-ANCA; however, none of them had features of concomitant connective tissue disease.

Nowadays, it is recognised that about half of the cases of IRF may be a symptom of a recently discovered, clinically heterogeneous immunoglobulin G4-related disease (IgG4-RD) [8, 9]. It is an immune-mediated disease, hallmarked by several features: a tendency to form tumefactive lesions, a unique histopathologic pattern in affected organs, and elevated (in most cases, though not always) concentrations of IgG4 in plasma. It seems important to identify patients with IgG4-related RPF due to the risk of involvement of other organs, located outside the retroperitoneum, and due to the need for probably more aggressive immunosuppressive therapy in this group, compared to IRF [10]. Regrettably, the evaluation of IgG4 serum level was not technically feasible at our department.

According to the literature, CS are the mainstay of treatment of IRF. The suggested initial dose of prednisone 
usually ranges from 30 to $60 \mathrm{mg}$ (0.5 to $1 \mathrm{mg} / \mathrm{kg}$ ) daily [2, 11-13]. Nowadays, successful attempts are also made to use alternative immunosuppressant drugs both in combination with CS and as monotherapy. In our study, the initial CS dosage was slightly lower than usually recommended, but four patients received AZA and one patient received intravenous CS pulses, additionally. In our opinion, it is reasonable to use such a combination to minimize the risk of side effects associated with long-term intake of high doses of CS. Several small case series have demonstrated the efficacy and safety of AZA in the management of IRF. E.g., promising results were presented by Marcolongo et al. [14]: the combination of prednisone and AZA proved to be effective as first-line therapy and safer than the combination of prednisone and cyclophosphamide. In a retrospective analysis performed by Průcha et al. [15], AZA with CS were applied in 26 patients with RPF: clinical and radiological improvement was observed in all cases; however, two patients experienced disease exacerbation after treatment discontinuation. AZA has also been successfully used for remission maintenance [16]. In a study conducted by Moroni et al. [17], patients were divided into three groups: treated with CS + ureterolysis (group 1), CS + AZA (group 2), and CS + tamoxifen (group 3). In most patients, each of the three different approaches restored renal function and significantly reduced the fibrotic mass. These results suggest that combined therapy should be considered as the first-line strategy, especially in severe cases. Other possible options include methotrexate, mycophenolate mofetil, tamoxifen, cyclophosphamide, cyclosporine and biological agents [18-20]. There is also no consensus regarding the duration of treatment of IRF, although most authors suggest maintaining the therapy for 1 to 3 years [11-13]. Due to the lack of large prospective studies, recommendations for the management of RPF are yet to be established.

In summary, we present the clinical features and outcomes of five patients with RPF. We believe that increasing awareness of this disease and collaboration between clinicians of various specialities are the key to the early diagnosis of RPF and prevention of its potentially irreversible complications.

The authors declare no conflict of interest.

\section{References}

1. Vaglio A, Salvarani C, Buzio C. Retroperitoneal fibrosis. Lancet 2006; 367: 241-251.

2. Alberici F, Palmisano A, Urban ML, et al. Methotrexate plus prednisone in patients with relapsing idiopathic retroperitoneal fibrosis. Ann Rheum Dis 2013; 72: 1584-1586.
3. Yachoui R, Sehgal R, Carmichael B. Idiopathic retroperitoneal fibrosis: clinicopathologic features and outcome analysis. Clin Rheumatol 2016; 35: 401-407.

4. Minocha P, Setia A. Presentation of idiopathic retroperitoneal fibrosis at a young age: A rare case report. Intractable Rare Dis Res 2016; 5: 294-296.

5. Wolski H, Seremak-Mrozikiewicz A, Woyciechowska A, et al. Retroperitoneal fibrosis - case report and literature review. Ginekol Pol 2015; 86: 867-871.

6. Corradi D, Maestri R, Palmisano A, et al. Idiopathic retroperitoneal fibrosis: clinicopathologic features and differential diagnosis. Kidney Int 2007; 72: 742-753.

7. Alberti C. Revisiting the retroperitoneal fibroses: Are there any significant news about their aetiopathogenesis and diagnostic approaches? Eur Rev Med Pharmacol Sci 2012; 16: 1078-1094.

8. Khosroshahi A, Carruthers MN, Stone JH, et al. Rethinking Ormond's Disease. Medicine 2013; 92: 82-91.

9. Zen Y, Onodera M, Inoue D, et al. Retroperitoneal Fibrosis: A Clinicopathologic Study With Respect to Immunoglobulin G4. Am J Surg Pathol 2009; 33: 1833-1839.

10. Koo BS, Koh YW, Hong S, et al. Clinicopathologic characteristics of IgG4-related retroperitoneal fibrosis among patients initially diagnosed as having idiopathic retroperitoneal fibrosis. Mod Rheumatol 2014; 7595: 1-5.

11. Fry AC, Singh S, Gunda SS, et al. Successful use of steroids and ureteric stents in 24 patients with idiopathic retroperitoneal fibrosis: A retrospective study. Nephron Clin Pract 2008; 108 : 213-220.

12. Kardar AH, Kattan S, Lindstedt E, et al. Steroid therapy for idiopathic retroperitoneal fibrosis: dose and duration. J Urol 2002; 168: 550-555.

13. van Bommel EFH, Siemes C, Hak LE, et al. Long-Term Renal and Patient Outcome in Idiopathic Retroperitoneal Fibrosis Treated With Prednisone. Am J Kidney Dis 2007; 49: 615-625.

14. Marcolongo R, Tavolini IM, Laveder F, et al. Immunosuppressive therapy for idiopathic retroperitoneal fibrosis: a retrospective analysis of 26 cases. Am J Med 2004; 116: 194-197.

15. Průcha M, Kolombo I, Štádler P. Combination of steroids and azathioprine in the treatment of Ormond's disease - a single centre retrospective analysis. Prague Med Rep 2016; 117: 34-41.

16. Binder $\mathrm{M}$, Uhl $\mathrm{M}$, Wiech T, et al. Cyclophosphamide is a highly effective and safe induction therapy in chronic periaortitis: a long-term follow-up of 35 patients with chronic periaortitis. Ann Rheum Dis 2012; 71: 311-312.

17. Moroni G, Gallelli B, Banfi G, et al. Long-term outcome of idiopathic retroperitoneal fibrosis treated with surgical and/ or medical approaches. Nephrol Dial Transplant 2006; 21: 2485-2490.

18. Kermani TA, Crowson CS, Achenbach SJ, et al. Idiopathic retroperitoneal fibrosis: a retrospective review of clinical presentation, treatment, and outcomes. Mayo Clin Proc 2011; 86: 297-303.

19. Urban ML, Palmisano A, Nicastro M, et al. Idiopathic and secondary forms of retroperitoneal fibrosis: A diagnostic approach. Rev Med Interne 2015; 36: 15-21.

20. Shiber S, Eliakim-Raz N, Yair M. Retroperitoneal fibrosis: case series of five patients and review of the literature. Rev Bras Reumatol 2016; 56: 101-104. 